\title{
Enfermedades de la espinaca (Spinacia oleracea L.) en Cota (Cundinamarca) y manejo del mildeo velloso (Peronospora farinosa, Byford)
}

\section{Spinach (Spinacia oleracea L.) diseases in Cota (Cundinamarca) and control of downy mildew (Peronospora farinosa, Byford)}
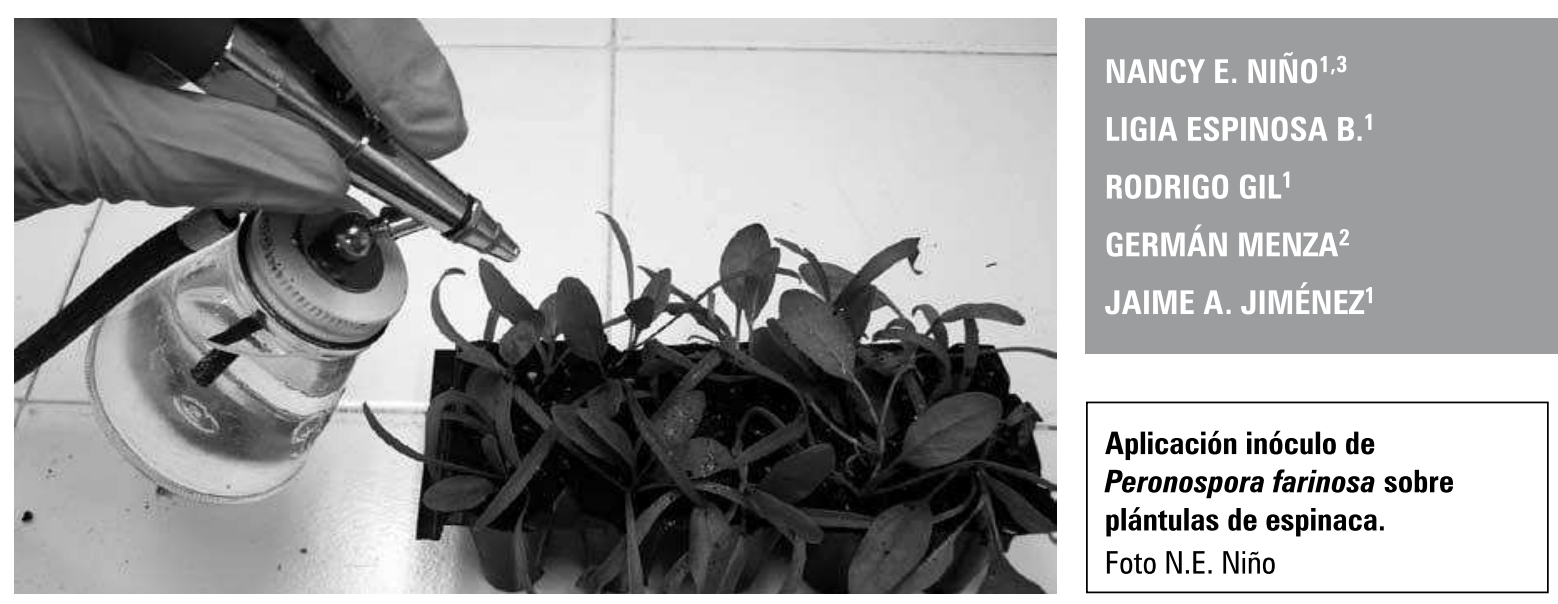

\section{RESUMEN}

Se identificaron las principales enfermedades en el cultivo de espinaca en Cota (Cundinamarca), considerando tres etapas del ciclo vegetativo: germinación, desarrollo de hojas y cosecha. Durante la germinación, la enfermedad con mayor incidencia fue "Damping-off" o volcamiento, causado por el complejo Fusarium oxysporum, Pythium sp. y Rhizoctonia solani (8,19\%). Durante el desarrollo de hojas, el mildeo velloso (Peronospora farinosa) tuvo la mayor incidencia (32,05\%), seguida por hongos foliares: Cladosporium sp., Alternaria sp. y Stemphylium sp. $(5,91 \%)$ y pudrición blanda de corona, causada por Erwinia carotovora $(\leq 2 \%)$. Durante la cosecha, se presentaron manchas foliares causadas por Pseudomonas syringae (5,13\%) y la incidencia del mildeo velloso disminuyó a 10,8\%. Siendo el mildeo velloso la principal enfermedad en espinaca, se evaluó el efecto preventivo de tres fungicidas químicos (Metalaxil+Mancozeb, Propamocarb y Fosetil Aluminio), tres productos botánicos comerciales (hidrolatos de manzanilla, ajo-ají y cola de caballo) y tres productos biológicos comerciales (Trichoderma harzianum, T. lignorum y Bacillus subtilis) sobre el patógeno en condiciones controladas, utilizando una escala de severidad (0-4). Los productos químicos inhibieron en 100\% la germinación de esporangios de $P$. farinosa y la expresión de la enfermedad en plántulas. Los tres hidrolatos y los hongos antagonistas presentaron poco efecto inhibitorio sobre $P$. farinosa, mientras que B. subtilis, inhibió en $92 \%$ la germinación de esporangios y presentó un valor bajo $(1,8)$, en la escala de severidad, mostrando buen potencial para el control de este patógeno.

\footnotetext{
Centro de Investigaciones y Asesorías Agroindustriales, Universidad de Bogotá Jorge Tadeo Lozano, Chía (Colombia).

2 Programa Microbiología Agrícola y Veterinaria, Facultad de Ciencias, Pontificia Universidad Javeriana, Bogotá (Colombia).

3 Autora para correspondencia.nancynino@hotmail.com
} 


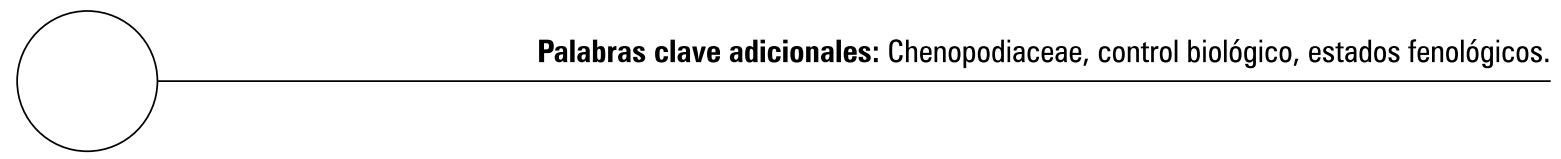

\section{ABSTRACT}

There were identified the principle spinach diseases in Cota, Cundinamarca, taking into account three stages of the vegetative cycle: germination, leaf development and crop harvest. During the germination, the major incident disease was "Damping-off", caused by the complex Fusarium oxysporum, Pythium sp. and Rhizoctonia solani $(8.19 \%)$. During the leaf development, downy mildew (Peronospora farinosa) had the major incidence (32.05\%), followed by foliage fungi Cladosporium sp., Alternaria sp. and Stemphylium sp. (5.91\%) and soft rotting of crown, caused by Erwinia carotovora ( $>2 \%)$. During the crop harvest, appeared leaves spots caused by Pseudomonas syringae (5.13\%) and downy mildew incidence were reduced down to $10.18 \%$. Being downy mildew the main disease in spinach, there was evaluated the preventive effect of three chemical fungicides (Metalaxil+Mancozeb, Propamocarb and Fosetil Aluminio), three botanical products (chamomile, garlic-chili and tail of horse), and three biological products (Trichoderma harzianum, T. lignorum and Bacillus subtilis) on the pathogenic fungi in controlled conditions, using a severity scale (0-4). The chemical products inhibited $100 \%$ germination of $P$. farinos $a$ sporangia and expression of the disease on seedlings. The three botanical products and fungi antagonists presented little inhibitory effect on $P$. farinosa, meanwhile B. subtilis diminished on $92 \%$ sporangia germination and presented a low value in the severity scale (1.8), proving to be of high potential for downy mildew control.

Additional key words: Chenopodiaceae, biological control, phenological stages.

Fecha de recepción: 13-10-2009

Aprobado para publicación: 30-11-2009
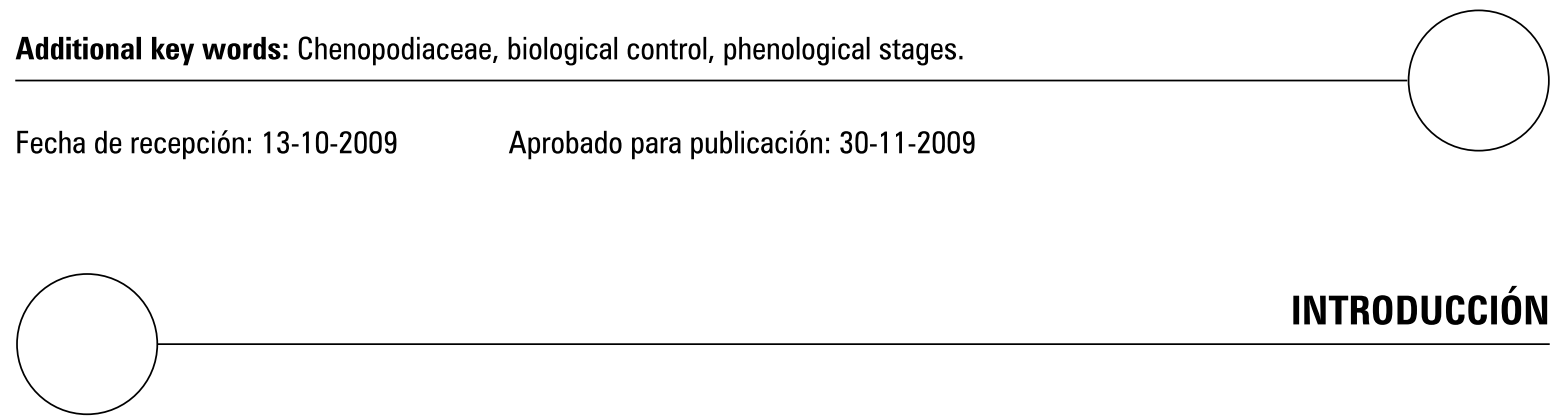

La espinaca (Spinacea oleracea L.) es una hortaliza perteneciente a la familia Chenopodiaceae, es originaria del centro de Asia, donde se ha cultivado por más de 1.300 años (Corell, 1994). Entre sus cualidades nutricionales se destacan su contenido de agua, vitaminas B1, B2 y C, y minerales (calcio, fósforo y hierro) (FAO, 2009). China es el mayor productor de espinaca a nivel mundial, con el $90 \%$, seguido por Estados Unidos $(7 \%)$ y Japón (3\%).

A nivel mundial, Estados Unidos, reporta el mildeo velloso (Peronospora farinosa) y la roya blanca (Albugo occidentalis) como las de mayor impor- tancia económica en espinaca. Adicionalmente los hongos: Colletotrichum dematium, Cladosporium macrocarpum, Alternaria spp. y Stemphyllium botryosum, causando manchas foliares. En plántulas, Damping-off causado por: Pythium sp., Fusarium oxysporum y Rhizoctonia solani; nematodos fitoparásitos como: Heterodera schacctii, H. trifolii, Meloidogyne incognita y Pratylenchus spp. Enfermedades bacteriales: Pseudomonas syringae y Erwinia carotovora; afectando hojas y corona en plantas. Así mismo, los virus del mosaico del pepino (CMV) y del amarillamiento occidental de la remolacha (BWYV) (Corell et al., 1994; Summer, 2000; Koike et al., 2001; Chaney et al., 2005). 
En Colombia, los principales departamentos productores de esta hortaliza son Cundinamarca, con una producción de 2.918 t, le sigue Antioquia con 504 t y, por último, Norte de Santander, con 192 t (Agronet, 2008). En la Sabana de Bogotá, el municipio con mayor cantidad de lotes dedicados a la siembra de espinaca es Cota, según censo agrícola del DANE (2006), en donde se siembran en promedio 140 ha anualmente.

Uno de los principales limitantes de su producción son las plagas y enfermedades, entre las cuales Guzmán (1986) reporta: mildeo velloso (Peronospora farinosa), Cladosporium sp., Pythium sp. y Alternaria sp. En la Sabana de Bogotá, el mildeo velloso es la principal enfermedad que afecta la espinaca, debido a las condiciones climáticas que favorecen su desarrollo y a que este patógeno puede sobrevivir en suelo y en semillas por largo tiempo. En el municipio de Cota la siembra de espinaca es continua y en muchas fincas no se hace rotación de cultivos, factor que además favorece la incidencia de la enfermedad (Chabur, 2008). Para su control se recomienda el uso de variedades resistentes y la aplicación preventiva de fungicidas (Brandenberger et al., 1994; Corell et al., 1994).

La demanda de alimentos libres de residuos de agroquímicos, ha llevado a la búsqueda de nuevas sustancias bioactivas, como los extractos vegetales y hongos antagonistas, los cuales tienen un menor impacto sobre el medio ambiente y la salud del hombre (Luchini, 2000; Alcalá de Marcano et al., 2005).

Los extractos de ajo y manzanilla, utilizados actualmente en el control de enfermedades de plantas, contienen sustancias volátiles inhibitorias del crecimiento microbiano (Naganawa et al., 1996; Cowan, 1999; Cerón et al., 1999; Scalia et al., 1999; Rodríguez, 1999). El ajo (Allium sativum L.) posee elementos azufrados como alicina, alina, cicloide de alicina y disulfuro de dialil con propiedades bactericidas y fungicidas (Naganawa et al., 1996; Bianchi et al., 1997; Obagwu y
Korsten, 2003; Curtis et al., 2004). La manzanilla (Matricaria chamomilla L.) contiene principalmente trans- $\beta$-farneseno y compuestos fenólicos como taninos (Mulinacci et al., 2000; Sokovic y van Griensven, 2006; Szoke et al., 2004). El Equisetum arvense (cola de caballo), contiene ácido silícico y fosfatidilcolina procedentes de la lecitina, compuesto que actúa como fortalecedor de la pared celular de las plantas (Abonos Superior, 2009). Así mismo los hongos antagonistas del género Trichoderma actúan como biocontroladores, por medio de la promoción del crecimiento vegetal, inducción de resistencia, competencia, micoparasitismo, antibiosis y producción de enzimas (Elad, 2000; Sid et al., 2003; McLean et al., 2005; Sarroco et al., 2006; Dubey et al., 2007). Se ha demostrado el efecto antagonista de Trichoderma sobre hongos fitopatógenos como: Rhizoctonia spp., Fusarium spp., Pythium spp., Botrytis spp., Phytophthora spp., entre otros. Algunas bacterias como Bacillus subtilis, Pseudomonas spp. y Serratia spp., actualmente son utilizadas como microorganismos antagonistas (Fernández-Larrea, 2001; Bernal et al., 2006; Agricultura orgánica, 2009).

Considerando que en Colombia la información disponible sobre el manejo de enfermedades en espinaca es limitada, en esta investigación se evaluaron diferentes alternativas de control sobre mildeo velloso, con productos químicos, botánicos y biológicos, disponibles comercialmente, con el fin de plantear alternativas de manejo para este patógeno.

\section{MATERIALES Y MÉTODOS}

\section{Identificación de las enfermedades}

La colecta de muestras de espinaca se realizó en las fincas El Alcalá, El Hoyo y San Ignacio, pertenecientes al municipio de Cota (Cundinamarca), ubicado a $4^{\circ} 49^{\prime} 05^{\prime \prime} \mathrm{N}, 7^{\circ} 07^{\prime} 20^{\prime \prime}$ W y a $2.547 \mathrm{msnm}$, con temperatura media de $13,7^{\circ} \mathrm{C}$ y un régimen de precipitación bimodal, con un promedio anual de $700 \mathrm{~mm}$. 
Se realizaron muestreos semanales durante un ciclo de producción, utilizando cuadros de 0,25 $\mathrm{m}^{2}$ en cada finca como unidades experimentales (20/finca). Los cuadros se ubicaron dentro de una grilla regular y en cada uno se registró el número de plantas, sus estados sanitario y de desarrollo. Las plantas con signos o síntomas de enfermedades se llevaron al laboratorio de fitopatología del Centro de Investigaciones y Asesorías Agroindustriales (CIAA) para su identificación.

Para este estudio, el ciclo de desarrollo de la espinaca se dividió en tres etapas, según las "Escalas BBCH" (Meier, 2001). La primera etapa comprendió desde la siembra de la semilla hasta la formación de hojas cotiledonales (0 a 10, escala $\mathrm{BBCH}$ ) y se denominó "germinación". La segunda etapa comprendió desde la aparición de las primeras hojas verdaderas hasta alcanzar $70 \%$ del diámetro esperado (11 a 37) y se denominó "desarrollo de hojas". La última etapa desde la formación del $70 \%$ de la roseta foliar, hasta su completo desarrollo (38 y 39), se denominó etapa de "cosecha" (Gil et al., 2007).

\section{Evaluación de estrategias de manejo de $P$. farinosa}

Teniendo en cuenta que $P$. farinosa fue la enfermedad de mayor porcentaje de incidencia en campo, se plantearon diferentes tratamientos para el manejo de la enfermedad, con el fin de evaluar su efecto sobre la germinación de esporangios y sobre la severidad de la enfermedad en plántulas de espinaca de 2 semanas de edad, en condiciones controladas, utilizando una escala de severidad de 0 a 4 (tabla 1).

Se evaluaron tres fungicidas químicos: dos productos utilizados por los Agricultores de espinaca en Cota: Metalaxil+Mancozeb y Propamocarb, y el fungicida Fosetil Aluminio, utilizado ampliamente en otros países como Estados Unidos, donde ya está registrado para este cultivo (Irish y Corell, 2004; Hochmuth et al., 2005), tres productos botánicos: manzanilla, ajo-ají y cola de caballo, teniendo en cuenta que son los más utilizados para el control de hongos foliares, y tres productos biológicos comerciales registrados como antagonistas: Trichoderma harzianum, T. lignorum y Bacilus subtilis (tabla 2).

\section{Efecto de fungicidas sobre la germinación de esporangios de $P$. farinosa}

Para evaluar el efecto de los productos fungicidas sobre la germinación de esporangios, se prepararon soluciones de los fungicidas a la dosis comercialy en esta solución se lavaron hojas de espinaca con esporangios de $P$. farinosa provenientes de la

Tabla 1. Escala de severidad de $P$. farinosa, con base en el porcentaje de esporulación sobre hojas cotiledonales de espinaca.

\begin{tabular}{|c|c|c|}
$\begin{array}{c}\text { Escala } \\
\text { severidad }\end{array}$ & $\begin{array}{c}\text { \% de esporulación P. farinosa en hojas cotiledonales } \\
\text { Plántulas sin síntomas de enfermedad }\end{array}$ & $\begin{array}{c}\text { Hoja cotiledonal con 100\% de esporulación } \\
\text { (4 en escala de severidad) }\end{array}$ \\
\hline 1 & $1-25 \%$ & \\
\hline 2 & $26-50 \%$ & \\
\hline 3 & $51-75 \%$ & \\
\hline 4 & $76-100 \%$ & \\
\hline
\end{tabular}


Tabla 2. Productos fungicidas evaluados sobre Peronospora farinosa de espinaca.

\begin{tabular}{|c|c|c|c|c|c|c|}
\hline Grupos & Tratamientos & Productos & Casa comercial & Ingrediente activo & Dosis & Lote \\
\hline Testigo & TO & Agua & - & - & - & - \\
\hline \multirow{3}{*}{ Botánicos } & $\mathrm{T} 1$ & Hidrolato de ajo-ají & Xplode & $\begin{array}{l}\text { Capsaicina 6\%-Disulfuro } \\
\text { de Alilo } 4 \%\end{array}$ & $1,0 \mathrm{~cm}^{3} \mathrm{~L}^{-1}$ & 88 \\
\hline & $\mathrm{T} 2$ & $\begin{array}{l}\text { Hidrolato de cola } \\
\text { de caballo }\end{array}$ & Abonos Superior & Cumarina - Equisetonina & $0,5 \mathrm{~cm}^{3} \mathrm{~L}^{-1}$ & 5584 \\
\hline & T3 & $\begin{array}{l}\text { Hidrolato de } \\
\text { manzanilla }\end{array}$ & Abonos Superior & $\begin{array}{l}\text { A. Salicílico, Fenoles, } \\
\text { Taninos, Flavonoides, etc. }\end{array}$ & $1,0 \mathrm{~cm}^{3} \mathrm{~L}^{-1}$ & 5476 \\
\hline \multirow{3}{*}{ Biológicos } & $\mathrm{T} 4$ & Laverlam & Laverlam & Bacillus subtilis & $0,5 \mathrm{~cm}^{3} \mathrm{~L}^{-1}$ & 108 \\
\hline & T5 & Foliguard sc & LST & Trichoderma harzianum & $0,5 \mathrm{~cm}^{3} \mathrm{~L}^{-1}$ & 558 \\
\hline & T6 & Mycobac wp & Laverlam & Trichoderma lignorum & $0,5 \mathrm{~cm}^{3} \mathrm{~L}^{-1}$ & 068043-1 \\
\hline \multirow{3}{*}{ Químicos } & $\mathrm{T} 7$ & Ridomil gold & Syngenta & Metalaxil+Mancozeb & $2,5 \mathrm{~cm}^{3} \mathrm{~L}^{-1}$ & SCAB9137 \\
\hline & T8 & Fosetal & Químicos Oma & Fosetil Aluminio & $2,5 \mathrm{~cm}^{3} \mathrm{~L}^{-1}$ & 3479 \\
\hline & T9 & Previcur & Bayer & Propamocarb & $2,5 \mathrm{~cm}^{3} \mathrm{~L}^{-1}$ & RK0707012 \\
\hline
\end{tabular}

finca Alcalá del municipio de Cota, quedando los esporangios en la suspensión con fungicida. A los testigos se les lavaron las hojas con el patógeno en agua destilada. Como unidades experimentales, se utilizaron: cajas de petri plásticas de $4 \mathrm{~cm}$ de diámetro, con medio agar-agua. Con una micropipeta, se colocó una alícuota de $1 \mathrm{~mL}$ de la suspensión de esporangios de $P$. farinosa sobre cada caja de petri. Los tratamientos se replicaron cinco veces y se hicieron tres repeticiones en el tiempo.

Las cajas con esporangios se colocaron en nevera a $10^{\circ} \mathrm{C}$ y se mantuvieron durante $24 \mathrm{~h}$, luego se realizó el conteo de germinación, considerando un esporangio germinado, si el tubo germinativo presentaba una longitud del doble del diámetro del esporangio. Se hicieron tres conteos de esporangios germinados por caja y por conteo se observaron 100 esporangios.

\section{Preparación del inóculo para pruebas de eficacia fungicida}

El inóculo fue obtenido de hojas de espinaca colectadas directamente de campo de la finca Alcalá. En el laboratorio, las hojas con el patógeno se lavaron en agua y con ayuda de un pincel se realizaba la extracción del patógeno. La suspensión de esporangios se llevó a una concentración de $3 \times 10^{5}$ esporangios $/ \mathrm{mL}$, con la cámara de Neubauer.

\section{Pruebas de eficacia de fungicidas sobre plántulas de espinaca}

Se utilizaron plántulas de espinaca de la variedad Viroflay, cultivadas en rejillas con sustrato de turba de 2 semanas de edad, con dos pares de hojas verdaderas y se evaluó el efecto preventivo de los productos, aplicando primero los fungicidas y a las $24 \mathrm{~h}$ el inóculo de $P$. farinosa. Los fungicidas se prepararon en un volumen de 100 $\mathrm{mL}$, en la dosis comercial y en los testigos se aplicó agua sin fungicida. Los tratamientos y el inóculo se aplicaron con la ayuda de un microaspersor sobre las plántulas. Posteriormente las plántulas tratadas se llevaron a cámara húmeda a $20^{\circ} \mathrm{C}$ y $100 \% \mathrm{RH}$ por $24 \mathrm{~h}$ y se mantuvieron a $20^{\circ} \mathrm{C}$ y $80 \%$ HR durante $6 \mathrm{~d}$. Al sexto día, para inducir la esporulación, las plántulas se colocaron a $20^{\circ} \mathrm{C}$ y $100 \% \mathrm{HR}$ por $24 \mathrm{~h}$ (Brandenberger et al., 1991; Irish et al., 2003). Al séptimo día se realizaron evaluaciones cuantitativas utilizando la escala de severidad establecida. 


\section{Análisis de datos}

Para la evaluación de los tratamientos, se utilizó un diseño completamente aleatorizado con 10 réplicas y tres repeticiones en el tiempo. Se hizo análisis de varianza (Anova) y prueba de comparación Tukey, usando software versión 2.8.1; paquete Agricolae (Mendiburu, 2008).

\section{RESULTADOS Y DISCUSIÓN}

\section{Principales enfermedades en las tres fincas de Cota}

\section{Etapa de germinación}

Durante la germinación se presentó Dampingoff o volcamiento con una incidencia del 8,19\%. Esta enfermedad es causada por el complejo: Fusarium oxysporum, Pythium sp. y Rhizoctonia solani, lo cual concuerda con lo reportado por Corell et al. (1994) y Chaney et al. (2005), quienes encontraron que esta es una enfermedad común en las zonas productoras de espinaca en el mundo. La infección puede ocurrir antes de la emergencia de las plántulas y bajo condiciones favorables la mortalidad puede alcanzar el 100\%. La infección es favorecida por alta humedad, mal drenaje de los suelos y por una producción continua de espinaca (Agrios, 2005; Michelle et al., 2005).

Las plántulas de espinaca afectadas por Damping-off, presentaban pudrición de color marrón a negro en raíces y base del tallo. En síntomas avanzados, las raíces se pudren causando el colapso y muerte de las plántulas.

Corell et al. (1994) reportan la presencia de $P$. farinosa f. sp. spinaciae causando la muerte de plántulas de espinaca en zonas productoras de Estados Unidos, el cual puede persistir durante varios años en el suelo y sobrevivir en semillas. El hongo penetra en la planta por el tallo o raíces superficiales y luego, por los haces vasculares es trasladado a toda la planta. El manejo de esta enfermedad es preventivo y se basa en la siembra de variedades resistentes, ya que una vez penetra al tejido vegetal, no existe control químico efectivo. Esta enfermedad es favorecida por altas temperaturas y suelos ácidos (Agrios, 2005). Así mismo, las especies Pythium aphanidermatum (Edson) Fitzp., P. heterothallicum Campbell \& J. W. Hendrix y $P$. sylvaticum, se encuentran reportadas causando pudrición de raíz y pérdidas en espinaca en otros países productores (Sumner, 2000; AFS Spinach, 2009).

\section{Etapa de desarrollo de hojas}

Mildeo velloso. Esta fue la principal enfermedad, con una incidencia del 32,05\%. Los síntomas observados fueron manchas cloróticas de diferentes tamaños en el haz de las hojas, en el envés de la hoja se encuentran las estructuras del hongo compuestas por esporangios y esporangióforos de color gris, en hojas jóvenes eventualmente estas se distorsionan o se entorchan. La enfermedad es favorecida por condiciones de baja temperatura y alta humedad (Corell et al., 1994, 1998; Iris et al., 2007; Choi et al., 2007) (figura 1a y b).

El agente causal de esta enfermedad es Peronospora farinosa f. sp. spinaciae (Byford). Pertenece al reino Chromista, orden Peronosporales, familia Peronosporaceae y al género Peronospora (Choi et al., 2007), al que pertenecen los patógenos que ocasionan enfermedades conocidas como "mildeos vellosos"; son parásitos obligados, por tanto no crecen en medio artificial (Hall, 1996).

Este patógeno está reportado afectando especies de la familia Chenopodiaceae del género Beta, Spinacea y Chenopodium. P. farinosa solo afecta el género del cual es aislado y está subdividido en tres grupos de acuerdo a sus hospederos: $P$. farinosa f. sp. betae., $P$. farinosa f. sp. spinaciae y $P$. farinosa f. sp chenopodii (Danielsen y Ames, 2004).

Además del mildeo velloso, se encontraron otros hongos foliares como: Alternaria sp., Cladosporium sp. y Stemphylium sp., que causaron manchas 


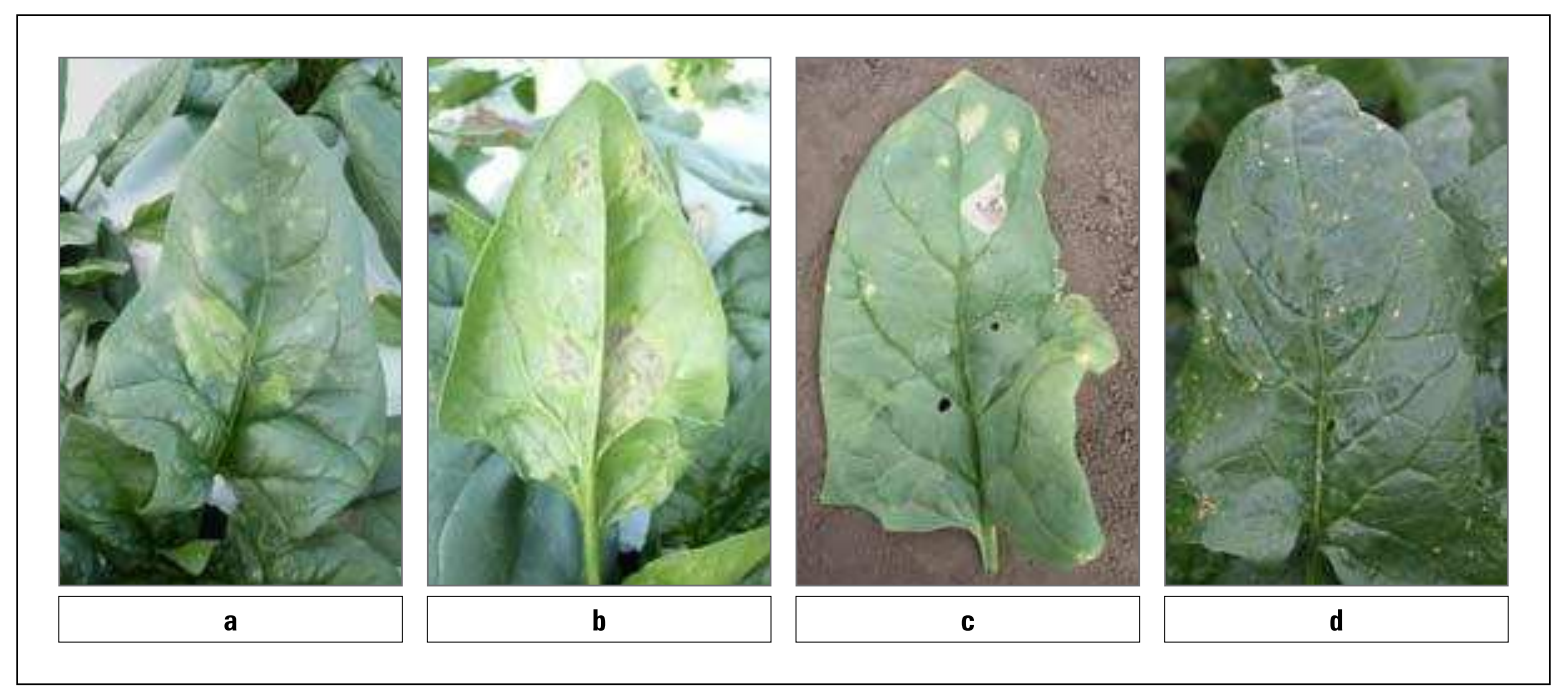

Figura 1. Síntomas de enfermedades en espinaca: a. Peronospora farinosa (haz), b. P. farinosa (envés), c. Alternaria sp., d. Stemphylium sp.

necróticas, los cuales presentaron una baja incidencia (5,91\%). Esto coincide con los reportes de Corell et al. (1994) y Koike et al. (2001), donde mencionan la baja incidencia de estos hongos en campo.

Alternaria. El síntoma de ataque por Alternaria se caracterizó por la presencia de manchas necróticas de color café claro, con la formación de anillos concéntricos característicos; generalmente afecta hojas inferiores y senescentes (figura 1c). El micelio y las conidias de Alternaria son de color oscuro, las conidias son alargadas, multicelulares, de forma periforme y forman cadenas simples o ramificadas.

Este género tiene especies cosmopolitas, con amplio rango de hospederos y pertenece a la familia Pleosporaceae de los Deuteromycetes (Ellis, 1971). Esta enfermedad está ampliamente distribuida en todo el mundo y se conoce también como tizón temprano, puede afectar hojas, tallos, flores y frutos de plantas anuales, principalmente hortalizas y ornamentales, aunque afecta algunos frutales y es difundido principalmente en zonas húmedas y de altas temperaturas. Cuando las plantas son sometidas a condiciones de estrés o a deficiencias nutricionales son más susceptibles a este patógeno. Sus conidias se diseminan por el viento o el agua y sobreviven en restos de tejidos enfermos. Así mismo, en condiciones de humedad y temperatura favorables, este patógeno puede causar daños importantes en hojas (Agrios, 2005).

Stemphylium. Los síntomas iniciales de Stemphylium en hojas consistieron en pequeñas manchas (1-3 mm de diámetro) de color gris que resaltan en hojas verdes. A medida que la enfermedad progresa, las manchas foliares se amplían y las hojas se van necrosando. El síntoma es similar al causado por Alternaria, aunque las manchas causadas por Stemphylium son más pequeñas y de forma irregular. Koike et al. (2001) denominaron el síntoma como: punto de la hoja (figura 1d).

Stemphylium pertenece a la familia Pleosporaceae de los Deuteromycetes (Ellis, 1971). Afecta principalmente hojas y su ciclo es similar a otros Deuteromycetes, sin embargo existe una amplia variabilidad entre hospederos, cuando se desarrolla bajo diferentes condiciones ambientales (Agrios, 2005).

Koike et al. (2001) reportan la especie S. botryosum como agente causal de mancha de la hoja en 
espinaca. En 2006, Hernández-Perez y du Toit, en una evaluación realizada en semillas de espinaca producidas en Estados Unidos, Dinamarca, y Nueva Zelanda, reportan S. botryosum con una incidencia del 29,1\%, y Cladosporium variabile con un $1,8 \%$, determinando la importancia de Stemphylium en semillas.

Cladosporium. Los síntomas de este hongo se caracterizaron por la formación de pequeñas manchas (1-2 $\mathrm{mm}$ ), de color café y de forma irregular sobre las hojas y se diferencia de los síntomas causados por Stemphylium, porque las estructuras del hongo (micelio y esporangios), son de color verde oscuro, en el centro de las manchas, así mismo esta enfermedad tiene baja incidencia en este cultivo según lo reportado a nivel mundial (Koike et al., 2001).

Cladosporium es un hongo cosmopolita, pertenece a los Deuteromycetes (Ellis, 1971) y la mayoría de especies son de hábito saprófito, sin embargo algunas son causantes de manchas foliares en hortalizas y ornamentales.

\section{Etapa de cosecha}

En esta etapa, la incidencia de mildeo velloso disminuyó significativamente a un 10,18\%, debido principalmente al manejo químico realizado por los agricultores. Así mismo, se presentaron enfermedades bacterianas con una baja incidencia.

Erwinia carotovora. Esta bacteria presentó una incidencia menor de $2 \%$ y se observó al finalizar la etapa de desarrollo de hojas y en cosecha. El síntoma principal fue la pudrición blanda del tejido interno de la corona y raíces. Los síntomas iniciales de esta enfermedad, no son fáciles de identificar, debido a que las plantas no presentan cambios aéreos visibles y generalmente se detecta al momento de la cosecha. Esta especie además fue reportada en espinaca por Corell et al. (1994).

E. carotovora pertenece al reino Procariote, familia Enterobacteriaceae, es anaerobia facultativa. Esta especie se caracteriza por tener una fuerte actividad pectolítica, causando pudriciones blandas, debido a que libera enzimas que maceran los tejidos vegetales. Las enfermedades bacterianas causadas por Erwinia son de difícil manejo y este se basa en medidas preventivas y culturales como: uso de semillas y material vegetal sano, buen manejo del drenaje y del riego; así mismo el control químico no es muy eficiente para su control (Agrios, 2005).

Pseudomonas syringae. Se presentó en la etapa de desarrollo de hojas y en cosecha, alcanzando una incidencia del 5,13\%. Los síntomas iniciales consistieron en pequeñas manchas de forma irregular, de color marrón oscuro a negras sobre hojas. En hojas maduras el síntoma es más severo, ya que invade casi toda el área foliar. Según Chaney et al. (2005), el problema está asociado con riego por aspersión o por lluvia.

P. syringae pertenece al reino Procariote, familia Pseudomonadaceae. Es aeróbica, gram-negativa. Esta especie se encuentra reportada en una amplia variedad de hortalizas como lechuga y tomate, como agente causal de manchas foliares. Se caracteriza por la producción de pigmentos fluorescentes en medios de cultivo específicos, lo cual ayuda a su diagnóstico.

Las bacterias del género Pseudomonas permanecen sobre órganos sanos o infectados de las plantas perennes, sobre semillas, restos de plantas infectadas, sobre recipientes o herramientas contaminadas y en el suelo. Su diseminación se lleva a cabo principalmente por el agua y la penetración se lleva a cabo por medio de aberturas naturales o por heridas (Agrios, 2005).

\section{Evaluación de estrategias de manejo del mildeo velloso}

\section{Efecto de fungicidas sobre germinación de esporangios de $\boldsymbol{P}$. farinosa.}

Según el Anova y prueba de clasificación de Tukey $\left(\mathrm{F}=<2,2 \mathrm{e}^{-16} ; P \leq 0,05\right)$, no se encontraron 
diferencias significativas entre el testigo (T0) y el tratamiento T2 (hidrolato de cola de caballo) en los tres ensayos, además, los tratamientos T1, T2, T3 y T5 (hidrolato de ajo-ají, cola de caballo, manzanilla y $T$. harzianum) fueron similares entre sí. Los tratamientos T4, T7, T8 y T9 $(B$. subtilis, Metalaxyl+Mancozeb, Fosetil Aluminio y Propamocarb) no presentaron diferencias significativas entre sí, y tuvieron el mayor efecto inhibitorio sobre la germinación de los esporangios de $P$. farinosa (tabla 3).

\section{Efecto de fungicidas sobre $P$. farinosa en plántulas}

No se encontraron diferencias significativas entre los tratamientos T0, T1, T2, T3, T4, T5 y T6, presentando los valores más altos de la escala de severidad de la enfermedad. Los tratamientos Metalaxyl+Mancozeb, Fosetil Aluminio y Propamocarb, alcanzaron 100\% de eficacia inhibiendo la expresión de la enfermedad en plántulas de espinaca y no presentaron diferencias significativas entre sí (tabla 4).

El fungicida Metalaxyl+Mancozeb es utilizado ampliamente para el control del mildeo velloso de espinaca en fincas del municipio de Cota, en donde se realizan de una a tres aplicaciones en un solo ciclo del cultivo. Este fungicida sistémico, es absorbido por las raíces y transportado a toda la planta y como lo reporta Brandenberger et al. (1991), se aplica de manera preventiva en muchas áreas de siembra de este cultivo para reducir la propagación del patógeno. Por otro lado, para el control de mildeo velloso, en Cota, se utiliza oxicloruro de cobre y Propineb, según encuesta con los agricultores de Cota (datos no publicados), aunque generalmente no utilizan las dosis recomendadas.

Según lo reportado en AFS (2009), los productos a base de Metalaxyl y Fosetil Aluminio han demostrado ser eficientes para el control del mildeo velloso de espinaca, cuando son aplicados en los primeros síntomas de la enfermedad. Así mismo, las semillas que contienen Metalaxyl o Cymoxanil ayudan a proteger la planta de la aparición hasta la etapa de cotiledón contra mildeos. En Estados Unidos (Crop Profile for Spinach Seed, 2005; Chaney et al., 2005) y Nueva Zelanda (Walter, 2003), además, se encuentran registrados para el manejo de mildeo velloso los fungicidas: Cymoxanil (Curzate 60DF); Mefenoxam (Ridomil Gold EC); Chlorothalonil (Utilizados también en el control de Cladosporium y Stem-

Tabla 3. Efecto de nueve tratamientos fungicidas sobre la germinación de esporangios de Peronospora farinosa.

\begin{tabular}{|c|c|c|c|c|c|c|}
\hline \multirow{2}{*}{\multicolumn{2}{|c|}{ Tratamientos }} & Ensayo 1 & Ensayo 2 & Ensayo 3 & Promedio & \multirow{2}{*}{$\begin{array}{l}\text { \% de inhibición } \\
\text { de germinación }\end{array}$} \\
\hline & & No. Esporas & No. Esporas & No. Esporas & (3 ensayos) & \\
\hline TO & Testigo Agua & 38,33 a & 39,00 a & 37,33 a & 38,22 & - \\
\hline $\mathrm{T} 1$ & H. Ajo-ají & $16,33 \mathrm{~cd}$ & $20,88 b$ & $22,33 a b$ & 19,85 & 48,1 \\
\hline $\mathrm{T} 2$ & H. Cola caballo & $29,00 \mathrm{ab}$ & $28,00 \mathrm{ab}$ & $32,22 a b$ & 29,75 & 22,2 \\
\hline T3 & H. Manzanilla & 13,00 cde & $17,66 \mathrm{~b}$ & $31,33 a b$ & 20,66 & 45,9 \\
\hline T4 & B. subtilis & 3,22 ef & $2,44 \mathrm{C}$ & $2,66 \mathrm{c}$ & 2,77 & 92,8 \\
\hline T5 & T. harzianum & $21,55 \mathrm{bc}$ & $16,00 \mathrm{~b}$ & $31,22 a b$ & 22,92 & 40,0 \\
\hline T6 & T. lignorum & 8,77 def & $16,33 \mathrm{~b}$ & $18,88 \mathrm{~b}$ & 14,66 & 61,7 \\
\hline $\mathrm{T7}$ & Metalaxyl+Mancozeb & $0 \mathrm{f}$ & $0 \mathrm{c}$ & $0 \mathrm{c}$ & 0 & 100,0 \\
\hline T8 & Fosetil Aluminio & $0 f$ & $0 \mathrm{c}$ & $0 \mathrm{c}$ & 0 & 100,0 \\
\hline T9 & Propamocarb & $0 \mathrm{f}$ & $0 \mathrm{c}$ & $0 \mathrm{c}$ & 0 & 100,0 \\
\hline
\end{tabular}

Promedios con letras distintas indican diferencia significativa según la prueba de Tukey $(P \leq 0,05)$. 


\begin{tabular}{|c|c|c|c|c|c|}
\hline & \multirow{2}{*}{ Tratamientos } & Ensayo 1 & Ensayo 2 & Ensayo 3 & Promedio \\
\hline & & No. Esporas & No. Esporas & No. Esporas & (3 ensayos) \\
\hline TO & Testigo Agua & $2,6 \mathrm{a}$ & $2,5 \mathrm{a}$ & 2,5 a & 2,5 \\
\hline $\mathrm{T} 1$ & H. Ajo-ají & $1,8 \mathrm{ab}$ & 2,0 a & 3,1 a & 2,3 \\
\hline T2 & H. Cola caballo & $2,3 a b$ & $2,4 \mathrm{a}$ & 2,3 a & 2,3 \\
\hline T3 & H. Manzanilla & $2,0 a b$ & $2,2 \mathrm{a}$ & 2,0 a & 2,1 \\
\hline T4 & B. subtilis & $1,4 b$ & 1,6 a & 2,6 a & 1,8 \\
\hline T5 & T. harzianum & $2,3 a b$ & 2,2 a & 2,6 a & 2,4 \\
\hline T6 & T. lignorum & $2,0 a b$ & 2,6 a & 2,0 a & 2,2 \\
\hline T7 & Metalaxyl+Mancozeb & $0 \mathrm{C}$ & $0 \mathrm{~b}$ & $0 \mathrm{c}$ & 0 \\
\hline T8 & Fosetil Aluminio & $0 \mathrm{c}$ & $0 \mathrm{~b}$ & $0 \mathrm{c}$ & 0 \\
\hline T9 & Propamocarb & $0 \mathrm{c}$ & $0 \mathrm{~b}$ & $0 \mathrm{c}$ & 0 \\
\hline
\end{tabular}

Promedios con letras distintas indican diferencia significativa según la prueba de Tukey $(P \leq 0,05)$.

phylium); hidróxido de cobre (aplicado en semi1la); Fosetil Aluminio (Aliette WDG) y Azoxystrobin (Amistar o Quadris), aplicados de forma preventiva, lo cual soporta lo propuesto en esta investigación.

De acuerdo con los estudios de Naganawa et al. (1996) y Alcalá de Marcano et al. (2005), el extracto de ajo (Allium sativum L.) contiene sustancias supresoras y un alto contenido de bisulfuro de alipropilo y podría considerarse como una alternativa para ser incorporado en una estrategia de manejo de hongos como Phytophthora infestans, Sclerotium rolfsii y Thielaviopsis basicola. Sin embargo, en esta investigación el hidrolato de ajo-ají no presentó efecto significativo sobre la germinación de esporangios de $P$. farinosa, ni sobre la expresión de esta enfermedad en plántulas de espinaca, posiblemente debido a su hábito biótrofo $y$ en condiciones favorables del patógeno, el extracto no logra inhibir su entrada a las plantas.

Estudios in vitro de Lee (2003) y de Reyes y Rodríguez (2001) apoyan el uso de combinaciones de hidrolatos en el manejo de la enfermedades causadas por hongos como Sclerotium cepivorum; además encontraron que la mezcla de Matrica- ria chamomilla, Solanum nigra y Eucaliptos globulus, y la mezcla de Tagetes sp., Taraxacum officinale (diente de león) y Datura stramonium eran las más eficaces en el control de esclerocios de $S$. cepivorum, mientras que un tratamiento con Crotalaria juncela, Ruda graveolens y Malva sylvestris presentó buen control de la enfermedad. Es importante anotar que en esta investigación el hidrolato de manzanilla no fue eficiente para el control de $P$. farinosa, evaluado directamente en plántulas de espinaca, por tanto, es necesario evaluar el efecto de estos hidrolatos, antes de ser incorporados en un plan de manejo integrado en campo.

Según estudios realizados en Guatemala, por Miranda (1996), el extracto de cola de caballo (Equisetum arvense), presentó buena eficiencia en el control del Phytophthora infestans, causante de "la gota" del tomate; sin embargo en esta investigación, este extracto presentó bajos porcentajes de eficacia para el control de $P$. farinosa en plántulas de espinaca.

B. subtilis presentó mayor efecto sobre la germinación de esporangios de $P$. farinosa y sobre la expresión de la enfermedad en plántulas de espinaca. Según la ficha técnica de este producto, $B$. 
subtilis genera lipopéptidos que actúan sobre las membranas celulares de los patógenos, afectando esporas, tubos germinativos y micelio, además previene la adhesión, penetración e infección de la superficie foliar, creando una zona de inhibición en la hoja. Debido a la complejidad de su mecanismo de acción, es una herramienta ideal en los programas de manejo de la resistencia. Así mismo, Heins et al. (2006) hacen referencia a varios investigadores como: Sholberg et al. (1995); Swinburne et al. (1975); Singh y Deverall (1984); Ferreira et al. (1991) y Baker et al. (1983), quienes describen el uso de $B$. subtilis como agente de biocontrol de patógenos fúngicos de plantas, el cual produce antibióticos que contribuyen a esta actividad fungicida.

En este estudio se encontró que las cepas comerciales T. harzianum y T. lignorum evaluadas sobre $P$. farinosa, no presentaron un control significativo sobre el patógeno, sin embargo, estudios anteriores realizados en Colombia por Ávila y Velandia (1992) reportaron, bajo condiciones de campo, que una cepa de T. harzianum, aplicado mediante el mismo procedimiento empleado para los productos químicos, presentó un efecto antagónico importante sobre Sclerotinia en lechuga. Así mismo, estudios de Arias et al. (2007), en condiciones de campo, demostraron que T. harzianum DSM 14944 presentó un control deficiente sobre S. sclerotiorum en lechuga, lo cual demuestra que cada cepa tiene una especificidad competitiva para cada patógeno.

Algunas especies de Trichoderma actúan como biocontroladores por medio de diferentes mecanismos, sin embargo, siendo un organismo de suelo, posiblemente su efecto sobre patógenos foliares es limitado debido a que las condiciones de luz y temperatura pueden afectar la viabilidad de este antagonista (Cotes, 2000; Queiroz et al., 2004; Sarroco et al., 2006; Dubey et al., 2007).

\section{CONCLUSIONES}

- Los tres hidrolatos vegetales evaluados, presentaron poco efecto sobre la germinación de los esporangios de $P$. farinosa y sobre la severidad de la enfermedad en plántulas de espinaca en condiciones controladas.

- B. subtilis presentó un alto porcentaje de inhibición de la germinación de esporangios de $P$. farinosa, siendo similar a los tratamientos químicos, constituyéndose como una alternativa para el control de este patógeno.

- Los productos químicos Metalaxyl+Mancozeb, Fosetil Aluminio y Propamocarb inhibieron en un $100 \%$ la germinación de esporangios de $P$. farinosa.

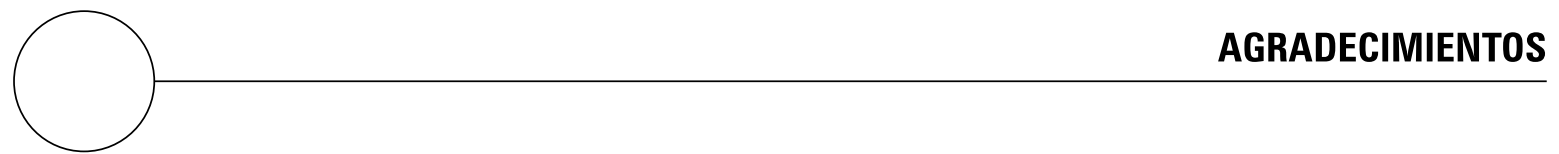

Los autores agradecen a las siguientes entidades, su apoyo y financiación: Ministerio de Agricultura y Desarrollo Rural, a la Asociación Hortifrutícola de Colombia (Asohofrucol), administradora del Fondo Nacional de Fomento Hortifrutícola, al Centro de Investigaciones y Asesorías Agroindustriales de la Universidad de Bogotá Jorge Tadeo Lozano, a las Alcaldías de Chía y Cota, y a las Cooperativas de agricultores Coophoticota y Ecomajuy. 


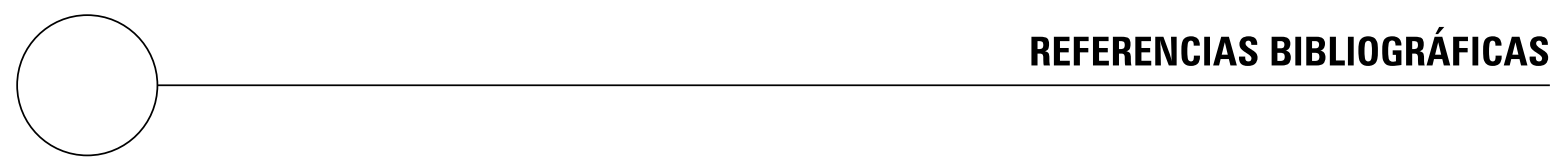

Abonos Superior. 2009. Ficha técnica productos hidrolatos. Bogotá.

Agricultura orgánica, 2009. Conceptos y usos del hongo Trichoderma sp. En http://www.controlbiologico. $\mathrm{com} / \mathrm{bp}$ trichobiol.htm; consulta: noviembre de 2009.

Agrios, G. N. 2005. Plant pathology. Department of Plant Pathology, University of Florida. 5a ed. Elsevier Academic, New York, NY.

Agronet. 2008. Análisis estadísticos. En: http://www. agronet.gov.co/; consulta: marzo de 2008.

Alcalá de Marcano, D.; N. Vargas y A. Pire. 2005. Efecto de extractos vegetales y fungicidas sintéticos sobre el crecimiento micelial in vitro de Sclerotium rolfsii y Thielaviopsis basicota. Rev. Fac. Agron. 22, 315-323.

Arias, L.A.; L.A. Cautiva; W. Piedrahita y B. Cháves. 2007. Evaluación de tres métodos de control del Moho blanco (Sclerotinia sclerotiorum (Lib.) de Bary) en lechuga (Lactuca sativa L.). Agron. Colomb. 25(1), 131-141.

Assured Food Standard (AFS) Spinach. 2009. Produce crop specific protocol spinach. En: http://www.assuredproduce.co.uk/resources/000/323/257/09Spinach final.pdf; consulta: noviembre de 2009.

Ávila C. y J. Velandia. 1992. Enfermedades de algunas especies hortícolas y su manejo. pp. 98-125. En: Primer Curso Nacional de Hortalizas de Clima Frío. C.I. Tabaitatá, Corpoica, Mosquera, Colombia.

Bernal, A.; I. Martínez; M. Díaz; L. Herrera y B. Martínez. 2006. Empleo de cepas de bacterias antagonistas en el control de Stemphylium solani Webber en tomate bajo cultivo protegido. Centro Agrícola 33(3), 37-40.

Bianchi, A.; A. Zambonelli y A. Zechini. 1997. Ultrastructural studies of the effects of Allium sativum on phytopathogenic fungi in vitro. Plant Dis. 81, 12411246.

Brandenberger, L.P.; J.C. Corell; T.E. Morelock y R.W. McNew. 1991. Identification and cultivar reactions to a new race (race 4) of Peronospora farinosa $\mathrm{f}$. sp. spinaciae on spinach in the United States. Plant Dis. $75,630-634$

Brandenberger, L.P.; J.C. Correll; T.E. Morelock y R.W. Mcnew. 1994. Characterization of resistance of spi- nach to white rust (Albugo occidentalis) and downy mildew (Peronospora farinosa f. sp. spinaciae). Phytopathol. 84(4), 431-437.

Brandenberger, L.P.; T.E. Morelock y J.C. Correll. 1992. Evaluation of spinach germplasm for resistance to a new race (Race 4) of Peronospora farinosa f. sp. Spinaciae. HortScience 27(10), 1118-1119.

Cerón, M.; E. Piña; C. Ávila; L. Gil; V. Numpaque; C. Villate y G. Llerena. 1999. Aplicaciones in vitro de los extractos de eucalipto, ajo, caléndula, hierbamora, manzanilla y ortiga en el control del hongo Sclerotium cepivorum. Fitopatol. Colomb. 23, 68-71.

Chabur, M.I. 2008. Identificación de razas y estudio preliminar de la diversidad genética de Peronospora farinosa f. sp. spinaciae agente causal del mildeo velloso en cultivos de espinaca en la sabana de Bogotá. Trabajo de grado. Microbiología Agrícola y Veterinaria, Facultad de Ciencias Básicas, Pontifica Universidad Javeriana, Bogotá.

Chaney, W.E.; M. Lestrange; M. Koike; R.F. Smith y S.A. Fennimore. 2005. Spinach UC IPM pest managementguidelines. En: http://www.ipm.ucdavis.edu/ PDF/PMG/; consulta: septiembre de 2009.

Choi, Y.J.; S.B. Hong y H.D. Shin. 2007. Re-consideration of Peronospora farinosa infecting Spinacia oleraceae as a distinct species, Peronospora effusa. Mycol. Res. $111,381-391$

Correll, J.C.; S.T. Koike; J. Sdhafer; J.M. Anders; B.M.J. Irish y T.E. Morelock. 1998. Two new races of the downy mildew pathogen (Peronospora farinosa $\mathrm{f}$. sp. spinaciae) of spinach in the United States (Abstract). Phytopathol. 88, S19.

Corell, J.C.; T.E. Morelock; M.C. Black; S.T. Koike; L.P. Brandenberger y F.J. Dainello. 1994. Economically important diseases of spinach. Plant Dis. 78(7), 653660.

Cotes, A. 2000. Utilización combinada de técnicas de pregerminación controlada de semillas y del agente de control biológico Trichoderma sp., para el control de patógenos radicales. pp. 137-141. En: Lópes, A. (ed.). I Curso-Taller Internacional Control Biológico, Componente Fundamental del Manejo Integrado de Plagas en una Agricultura Sostenible. Corpoica, Bogotá.

Cowan, M.M. 1999. Plant products as antiomicrobial agents. Clin. Microbiol. Rev. 10, 564-582. 
Crop Profile for Spinach Seed in Washington. 2005. Washington State University. College of Agricultural, Human \& Natural Resource Sciences. En: http:// www.tricity.wsu.edu/ cdaniels/profiles/SpinachSeedPM2.pdf; consulta: septiembre de 2009.

Curtis, H.; U. Noll; J. Stormann y A. Slusarenko. 2004. Broad-spectrum activity of the volatile phytoanticipin allicin in extracts of garlic (Allium sativum L.) against plant pathogenic bacteria, fungi and oomycetes. Physiol. Mol. Plant Path. 65, 79-89.

DANE (Departamento Administrativo Nacional de Estadística). 2006. Ministerio de Agricultura y Desarrollo Rural. Censo Hortícola, Sabana de Bogota.

Danielsen, S. y T. Ames. 2004. Mildew (Peronospora farinosa) of quinua (Chenopodium quinoa) in the Andian region. Benson Agriculture and Food Institute 110 B-49. Brigham Young University, Provo, UT.

Dubey, S.; M. Suresh y B. Singh. 2007. Evaluation of Trichoderma species against Fusarium oxysporum $\mathrm{f}$. sp. ciceris for integrated management of chickpea. Wilt. Biol. Control. 40, 118-127.

Elad, Y. 2000. Biological control of foliar pathogens by means of Trichoderma harzianum and potential modes of action. Crop Prot. 19, 709-714.

Ellis, M.B. 1971. Dematiaceous Hypomycetes. Commonwhealt Mycological Institute, Kew, Surrey, UK.

FAO. 2009. FAOSTAT. En: http://faostat.fao.org/faostat; consulta: diciembre de 2009.

Fernández-Larrea, V.O. 2001. Microorganismos antagonistas para el control fitosanitario. Manejo Integrado de Plagas 62, 96-100.

Gil, R.C.; D. Carrillo y J.G. Jiménez. 2007. Determinación de las principales plagas de la espinaca (Spinacia oleracea) en Cota, Colombia. Rev. Colomb. Entomol. 33(2), 124-128.

Guzmán, R. 1986. Producción y comercialización de la espinaca en la Sabana de Bogotá. Trabajo de grado. Facultad de Administración Agropecuaria, Universidad de la Salle, Bogotá.

Hall, G. S. 1996. Modern approaches to species concepts in downy mildews. Plant Pathol. 45, 1009-1026.

Heins, S.D.; D.C. Manker; D.R. Jimenez; R.J. McCoy; J.E. Orjala y P.G. Marrone. 2006. Nueva cepa de Bacillus destinada a luchar contra las enfermedades de las plantas y del gusano de las raíces del maíz. Agraquest. En: http://www.espatentes.com/ pdf/2268774_t3.pdf; consulta: octubre de 2009.

Hernández-Perez, P. y L.J. du Toit. 2006. Seedborne Cladosporium variabile and Stemphylium botryosum in spinach. Plant Dis. 90, 137-145.
Hochmuth, G.J.; C.S. Vavrina; W.M. Stall y S.M. Olson. 2005. Spinach production in Florida. Chapter 37. Horticultural Sciences Department, University of Florida, Gainesville, FL. pp. 325-328.

Irish, B.M.; J.C. Corell; S.T. Koike; J. Schafer y T. Morelock. 2003. Identification and cultivar reaction to three new races of the spinach downy mildew pathogen (Peronospora farinosa f. sp. spinaciae), from the United States and Europe. Plant Dis. 87,567-572.

Irish, B. y J. Correll. 2004. First report of Peronospora farinosa f. sp. spinaciae race 5 causing downy mildew of spinach in Florida. Plant Dis. 88, 567-572.

Koike, S.T.; D.M. Henderson y E.E. Butler. 2001. Leaf spot disease of spinach in California caused by Stemphylium. Plant Dis. 85, 126-130.

Lee, R. 2003. Reconversión de fincas a producción sostenible. Metodología interactiva. Universidad de Bogotá Jorge Tadeo Lozano, Bogotá.

Luchini, L.C. 2000. Agroquímicos: Comportamento no solo agua e planta. Summa Phytopatol. 26(1), 170-171.

McLean, K.; J. Swaminathan; C. Frampton; J. Hunt; H. Ridgway y A. Stewart. 2005. Effect of formulation on the rhizosphere competence and biocontrol ability of Trichoderma atroviride C52. Plant Pathol. 54, 212-218

Meier, U. 2001. Estadios de las plantas mono y dicotiledóneas. BBCH Monografía. Centro Federal de Investigaciones Biológicas para Agricultura y Silvicultura, Braunschweig. Blackwell WissenschaftsVerlag, Berlin.

Mendiburu, F. 2008. The agricolae package. statistical procedures for agricultural research. En: http://cranr.c3sl.ufpr.br/; consulta: septiembre de 2009.

Michelle, L.; S. Koike; J. Valencia y W. Chaney. 2005. Spinach production in California. Vegetable Research and Information Center. División of Agriculture and Natural Resources, University of California, Davis, CA.

Miranda, M.G. 1996. Evaluación de cuatro concentraciones de cola de caballo (Equisetum arvense), en el control de tizón tardío (Phytophthora infestans) en el cultivo de tomate (Lycopersicum esculentum), en San Gabriel, Solola. Facultad de Agronomía, Universidad de San Carlos, Guatemala.

Mulinacci, N.; A. Romani; P. Pinelli; F. Vincieri y D. Prucher. 2000. Characterization of Matricaria recutita L. flower extracts by HPLC-MS and HPLC-DAD analysis. Chromatographia 51, 731-737.

Naganawa, R.; N. Iwata; K. Ishikawa; H. Fukuda; T. Fujino y A. Suzuki. 1996. Inhibition of microbial 
growth by ajoene, a sulfur-containing compound derived from garlic. Appl. Environ. Microbiol. 62, 4238-4242.

Obagwu, J. y L. Korsten. 2003. Control of citrus green and blue molds with garlic extracts. Eur. J. Plant Pathol. 109, 221-225.

Queiroz, P.R.; M.C. Valadares-Inglis y P.W. Inglis. P. 2004. Survival in soil and detection of co-transformed Trichoderma harzianum by nested PCR. Pesq. Agropec. Bras. 39, 403-405.

Reyes, H.A. y B. Rodríguez. 2001. Evaluación de la combinación de hidrolatos en el control de Sclerotium cepivorum y Ditylenchus dipsaci en cebolla de bulbo (Allium cepa) en condiciones de vivero en Tunja, Boyacá. Trabajo de grado. Facultad de Ciencias Agropecuarias, Universidad Pedagógica y Tecnológica de Tunja, Colombia.

Rodríguez, H.R. 1999. Actividad de extractos vegetales sobre el crecimiento in vitro de Fusarium oxysporum $\mathrm{f}$. sp. Dianthi (Prill et Del), Alternaria solani (Ell \& Martin) y Rhizoctonia solani (Kuhn). Trabajo de grado. Facultad de Agronomía, Universidad Nacional de Colombia, Bogotá.

Sarroco, S.; L. Mikkelsen; M. Vergara; D. Jensen; M. Lübeck y G. Vannacci. 2006. Histopathological studies of sclerotia of phytopathogenic fungi parasitized by a GFP transformed Trichoderma virens antagonistic strain. Mycol. Res. 110, 179-187.
Scalia, S.; L. Giuffreda y P. Pallado. 1999. Analytical and preparative supercritical fluid extraction of chamomile flowers and its comparison with conventional methods. J. Pharm. Biomed. Anal. 21, 549-558.

Sid, A.; M. Ezziyyani; C. Pérez y M.E. Candela. 2003. Effect of chitin on biological control activity of Bacillus spp. and Trichoderma harzianum against root rot disease in pepper (Capsicum annuum) plants. Eur. J. Plant Pathol. 109, 633-637.

Sokovic, M. y L. van Griensven. 2006. Antimicrobial activity of essential oils and their components against the three major pathogens of the cultivated button mushroom, Agaricus bisporus. Eur. J. Plant Pathol. $116,211-224$

Sumner, R.D. 2000. Diseases of spinach (Spinacia oleracea L.). The American Phytopathological Society. En: http://www.apsnet.org/online/common/names/ spinach.asp; consulta: septiembre de 2009.

Szoke, E.; E. Máday; E. Tyihak; I. Kuzovkina y E. Lemberkovics. 2004. New terpenoids in cultivated and wild chamomile (in vivo and in vitro). J. Chromatogr. 800, 231-238.

Walter, M.; P. Harris-Virgin; W. Thomas, G. Tate; N.W. Waipara y G. Langford. 2003. Agrochemicals suitable for downy mildew control in New Zealand boysenberry production. Crop Prot. 23, 327- 333. 